\title{
NIVEL DE CONOCIMIENTO SOBRE EL ÁCIDO ÚRICO EN MÉDICOS, ENFERMERAS Y ESTUDIANTES DE DIFERENTES CENTROS DE SALUD Y UNIVERSIDADES DE SANTO DOMINGO, FEBRERO-ABRIL 2002.
}

\author{
Ricardo A. Jiménez Cocco* \\ José L. López Mena* \\ Dr. Jimmy Barranco*
}

\section{RESUMEN}

El ácido úrico (AU) se forma en el cuerpo como resultado del catabolismo de las purinas. Su concentración en la sangre puede elevarse produciendo inflamación del endotelio arterial, gota, tofos y cálculos renales. Se realizó un estudio prospectivo donde se entrevistaron 100 estudiantes de medicina; 50 de término y 50 de inicio, que asistieran a las universidades Instituto Tecnológico de Santo Domingo (INTEC), Universidad Autónoma de Santo Domingo (UASD) y Universidad Nacional Pedro Henríquez Ureña (UNPHU), también 50 médicos y 50 enfermeras de La Plaza de la Salud, Clínica Independencia, Centro Médico Alcántara \& Gonzáles y Hospital Salvador B. Gautier, para determinar el nivel de conocimiento que tienen sobre el AU y sus patologías.

Se encontró un déficit de conocimientos sobre el AU, el cual fue más notable en las enfermeras, donde el 52\% ignora la definición del AU. Un $65.5 \%$ de las personas afirmaron que el AU causa lesiones en la piel, sin

* Médico Endocrinólogo

** Estudiantes de Bio-Intec 
ser esto real. La muestra considera que alimentos como el tomate $(82.5 \%)$, la papa (4\%) y los productos lácteos (10\%) elevan los niveles sanguíneos de AU siendo esto falso. Los médicos y enfermeras deben mantenerse mejor informados y actualizarse con datos recientes para que no se siga propagando esta desinformación, porque el AU no es causante de las lesiones de las cuales se le acusa. Se recomienda realizar un estudio posterior a este para determinar los factores que han provocado el desconocimiento sobre el AU y sus patologías para realizar campañas y seminarios sobre el tema.

\section{PALABRAS CLAVES}

Catabolismo, purinas, ácido úrico, lesiones, proteína, tofos, artritis gotosa, cálculos renales, urea, hiperuricemia, orina; urato.

\section{Introducción}

$\mathrm{El}$ ácido úrico (AU) es un compuesto nitrogenado, blanco, inodoro e insípido, de fórmula $\mathrm{C}_{3} \mathrm{H}_{4} \mathrm{~N}_{4} \mathrm{O}_{3}$, que se forma en el cuerpo como resultado del metabolismo de las purinas. ${ }^{2}$ Está presente en pequeñas cantidades en la orina humana, y en cantidades mayores en la orina de las aves y reptiles. ${ }^{2}$ $\mathrm{El} \mathrm{AU}$ es poco soluble en agua e insoluble en alcohol y éter. Al calentarse forma urea, amoníaco y dióxido de carbono. ${ }^{1}$ El AU se produce a partir del amoniaco y por la degradación de nucleótidos procedentes de ácidos nucleicos; forma cristales que se acumulan regularmente en las articulaciones si no son desechadas por la orina. ${ }^{2}$

En el ser humano el AU se produce de la degradación de las purinas y por la desaminación de las proteínas; los uratos son la forma ionizada de AU y predominan en plasma, líquido extracelular y líquido sinovial. Aunque la síntesis de los nucleótidos de purina y su desintegración tienen lugar en todos los tejidos, el urato solo se sintetiza en los tejidos que contienen xantina oxidasa, sobre todo en el hígado e intesti- 
no delgado. ${ }^{3}$ Los niveles sanguíneos de AU varían en función de la edad y el sexo. La mayoría de los niños presenta una concentración plasmática de 3.0 a $4.0 \mathrm{mg} / \mathrm{dL}$. La concentración aumenta durante la pubertad en los varones, pero permanece baja en las mujeres hasta la menopausia, aunque no se comprende por completo la causa de esta variación en función del sexo, puede que sea en parte a la mayor excreción funcional de urato en las mujeres, como consecuencia de influencias hormonales; aunque la concentración de AU cambia en la etapa del embarazo, este se puede elevar hasta alcanzar los $6.5 \mathrm{mg} / \mathrm{dL}$ en el último trimestre del embarazo. ${ }^{3.4}$

Los valores plasmáticos de urato en varones adultos y mujeres premenopáusicas son de 7.0 y $6.0 \mathrm{mg} / \mathrm{dL}$ respectivamente. En los adultos, las concentraciones se elevan de forma estable con el tiempo y varían en función de la altura, el peso corporal, la tensión arterial, la función renal y el consumo de alcohol. ${ }^{3}$ En un sujeto normal sometido a un régimen pobre en purinas, los uratos se excretan en $70 \%$ por vía renal y el resto es eliminado a través del intestino; normalmente $98 \%$ del AU filtrado se reabsorbe, el restante $2 \%$ representa 10 $20 \%$ del total excretado, el restante $80-90 \%$ es eliminado por secreción tubular. ${ }^{4}$ Después de la menopausia, los valores en mujeres se elevan hasta alcanzar aproximadamente los de los varones. ${ }^{3}$ Una patología del AU es la hiperuricemia, que se puede definir como la concentración plasmática de urato mayor de $7.0 \mathrm{mg} / \mathrm{dL}$. La hiperuricemia se debe, el $10 \%$ de las veces, al aumento de la producción de AU y el $90 \%$ se debe a la disminución de la excreción de AU. ${ }^{5}$

Otra patología del AU es la artritis gotosa, también conocida como gota. Es una enfermedad reumática producida por el depósito de cristales de urato en algunos tejidos del organismo como son las articulaciones, los huesos, la piel y los riñones. ${ }^{6}$ Para que una persona sufra de la artritis gotosa debe 
tener Hiperuricemia por varios años o deficiencia renal crónica. ${ }^{2}$ Es causado también por la alta producción de AU, por la pobre eliminación del mismo, el consumo de alcohol excesivamente, estrés emocional, otra enfermedad y alguna lesión significativa o intervención quirúrgica. ${ }^{8}$

La gota se clasifica según la causa de la hiperuricemia, hablamos de gota primaria (90\% de los casos) cuando la causa de la hiperuricemia es una alteración genética y se habla de gota secundaria ( $10 \%$ de los casos) cuando la hiperuricemia es causa de otras enfermedades o la administración prolongada de fármacos. ${ }^{9}$

También se puede clasificar según la excreción renal del AU. Se han propuesto tres métodos para clasificar a los pacientes con gota según su excreción renal de AU: la uricuria (24hUur), el aclaramiento de AU (Cur) y la excreción de AU por volumen de filtrado glomerular (EurFG). La medición de 24hUur es la más empleada, aunque los otros dos también son usados. ${ }^{10}$

El tratamiento indicado para esta enfermedad es una dieta baja en purinas, suprimiendo las bebidas alcohólicas y limitando las carnes. Es recomendable comer muchas frutas y alimentos que contengan hidratos de carbono, beber más de 2 litros de agua diario y mucho reposo, porque muchas veces la artritis gotosa puede ser causada por estrés u obesidad. También es recomendable tomar medicamentos como antiinflamatorios y otros medicamentos que eleven el drenaje de los deshechos metabólicos. ${ }^{11-15}$

Es importante que la población tenga los conocimientos necesarios sobre el AU y las diferentes enfermedades que causa la elevación de la concentración del AU en el cuerpo. Las personas tienden a culpar al $\mathrm{AU}$ por sus lesiones en la piel. Es necesario que la gente esté conciente de qué es el AU, 
cuáles son sus síntomas y efectos en el cuerpo, cómo se manifiesta, qué enfermedades produce, de manera que no den falsas justificaciones de sus lesiones.

\section{Materiales y Métodos}

En este estudio prospectivo fue empleada la entrevista, instrumento principal del trabajo porque permitió determinar el nivel de conocimiento que tenía la muestra sobre el AU (A1). Por motivos de ética se mantuvo en anonimato el lugar de procedencia de la información tabulada, por lo que no es posible realizar comparaciones entre los niveles de conocimiento de los distintos centros.

La muestra tomada fue de 200 personas, elegidas al azar, con la única condición que tuvieran unos minutos para llenar la encuesta; de éstas 200 personas, 50 fueron médicos (MD), 50 enfermeras (EF), 50 estudiantes de término de medicina (ET) y 50 estudiantes de medicina que estuvieran iniciando la carrera (EI).

Fueron separados en estos grupos para poder comparar los resultados obtenidos con mayor facilidad, y para comprobar la falta de conocimiento que tienen estos grupos sobre el AU.

Existe una gran diferencia de edades por ser muestras diferentes, pero oscilaban entre los 17 a 50 años de edad. El género no fue considerado como criterio de exclusión-inclusión ya que el género no influye en los conocimimentos de las personas.

La muestra fue tomada en diversas instituciones públicas y privadas; estas fueron: La Plaza de la Salud, Clínica Independencia, Centro Médico Alcántara \& Gonzáles, Hospital Salvador B. Gautier, Instituto Tecnológico de Santo Domingo, Universidad Autónoma de Santo Domingo y Universidad Nacional Pedro Henríquez Ureña, todos ubicados en el Distrito Nacional de la República Dominicana. 


\section{Resultados y Discusión}

De 200 personas entrevistadas, $135(67.5 \%)$ fueron del género femenino y $65(32.5 \%)$ de género masculino. El $65 \%$ (130/200) de las personas sabían lo que era el AU; pero de estos resultados se debe tener en cuenta que los estudiantes de inicio tenían una referencia reciente sobre el AU, lo cual pudo haber alterado los resultados de esta variable y de este modo el $74 \%(37 / 50)$ respondieron correctamente. El resto de las personas $(35 \%)$ no sabían lo que era el AU o tenían una idea muy vaga del significado. Algunos de los médicos se le hacia complicado definir el AU ya que no se acordaban del significado, y por lo tanto contestaban incorrectamente a la pregunta. Las enfermeras, que obtuvieron un $48 \%$ (24/50), no sabían lo que era exactamente y si se acordaban del significado, lo empleaban incorrectamente.

Gráfica No. 1: Conocimientos básicos de ácido úrico en médicos, enfermeras y estudiantes.

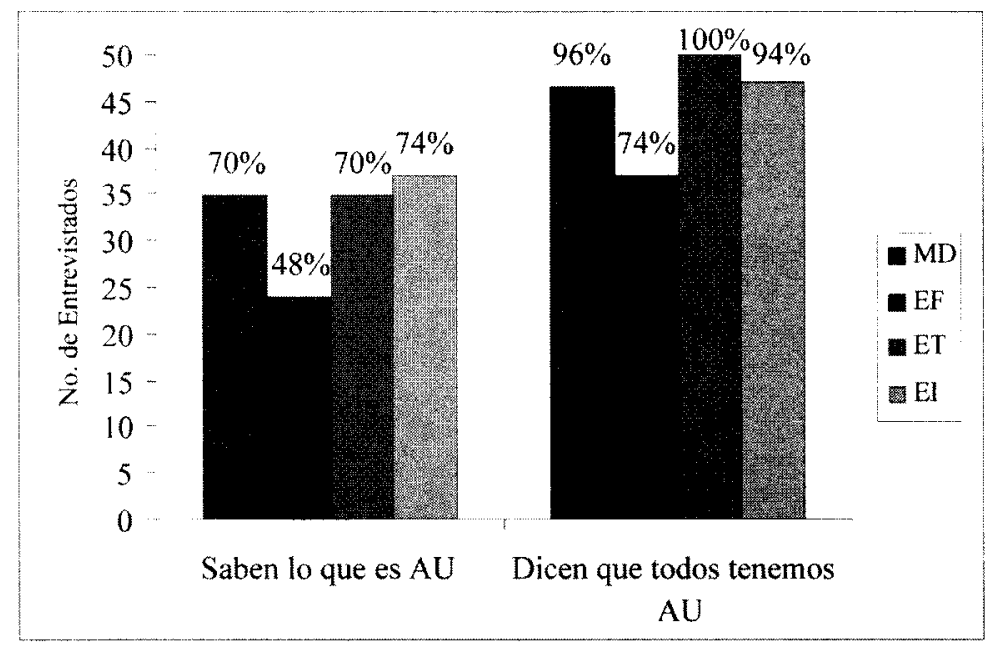

Fuente: Barranco, Jiméncz, Lópcz. Bio-Intec XLIII 
En la variable siguiente, “'Todos tenemos ácido úrico en el cuerpo?", la mayoría de los Médicos, Estudiantes de termino y Estudiantes de inicio acertaron al decir que sí; en cambio las enfermeras no sabían muy bien la respuesta a esta variable ya que sólo un 74\% (37/50) respondió que sí. Si el AU es el catabolismo de las purinas y proteínas, es lógico que todos tengamos AU en el organismo; ${ }^{1.3}$ en muchas ocasiones las/os enfermeras/os respondían correctamente a la variable anterior, pero incorrectamente en esta, contradiciéndose ellas mismas y demostrando la falta de educación que tenían. Un $66 \%$ (132/200) considera que el AU causa lesiones en la piel, como vesículas en las manos, pie de atleta, descamación de las palmas e inflamación de los pies, pero de estas la única que se acerca a lo correcto es inflamación en los pies, ya que es un síntoma de la artritis gotosa, enfermedad causada por la alta concentración de AU en la sangre.

Gráfica No. 2: Relación entre el ácido úrico y las lesiones en la piel.

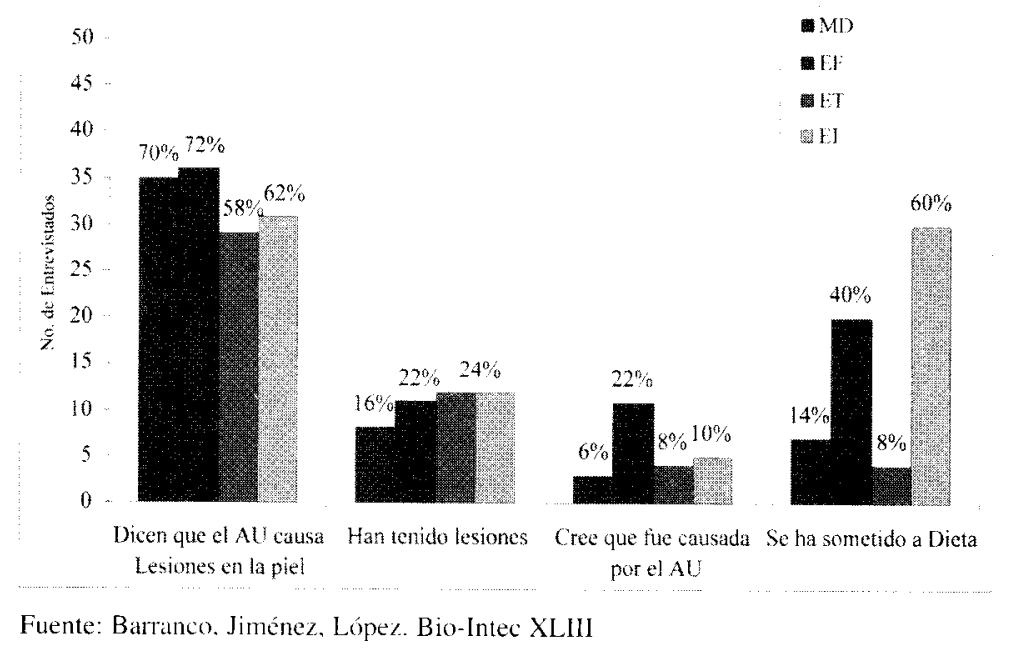


El $72 \%(36 / 50)$ de las enfermeras respondieron que el AU sí causa lesiones, en cambio, un 58\% (29/50) de los estudiantes de término respondió que sí, lo que indica que las generaciones de futuros médicos tienen mejor conocimiento sobre el $\mathrm{AU}$, aunque persiste la desinformación. Sólo un 39.5\% (79/200) dijo que el AU causa inflamación en los pies, esta lesión fue seleccionada en su mayoría por las enfermeras (48\%), pero los que destacaron la inflamación arterial fueron los médicos $(30 \%)$ y los estudiantes de término (menos del 25\%), lo que indicó la carencia de conocimiento del resto de la muestra. Un 26\% (52/200) de las personas piensan que el AU causa vesículas entre los nudillos, un $20.5 \%(41 / 200)$ dice que el AU causa pie de atleta y un $46.5 \%$ (93/200) dice que el AU causa descamación en las manos. Ninguno de estos trastornos es producto de la elevada concentración de $\mathrm{AU}$, ya que el $\mathrm{AU}$ no está asociado con ningún tipo de dermatitis. ${ }^{3}$ El 20\% (40/200) de las personas han tenidos lesiones como las mencionadas anteriormente, y el $15 \%$ (6/40), del $20 \%$ previamente dicho, dicen que fue causado por el AU. Todas las enfermeras que dijeron haber tenido estas lesiones aseguran que fue causado por el alto nivel de AU en la sangre, pero es muy difícil que una mujer sufra de hiperuricemia ya que esta patología esta ligada al cromosoma $\mathrm{X}$ y para que lo contraiga los dos cromosomas $\mathrm{X}$ de la mujer deben estar alterados. ${ }^{7}$ La única excepción es que la mujer esté en su último trimestre del embarazo o esté en la menopausia. ${ }^{4,9}$ De $8(16 \%)$ médicos lesionados, $3(37.5 \%)$ consideran que fue causado por el AU. 
Gráfica No. 3: Alimentos seleccionados por los médicos, enfermeras y estudiantes.

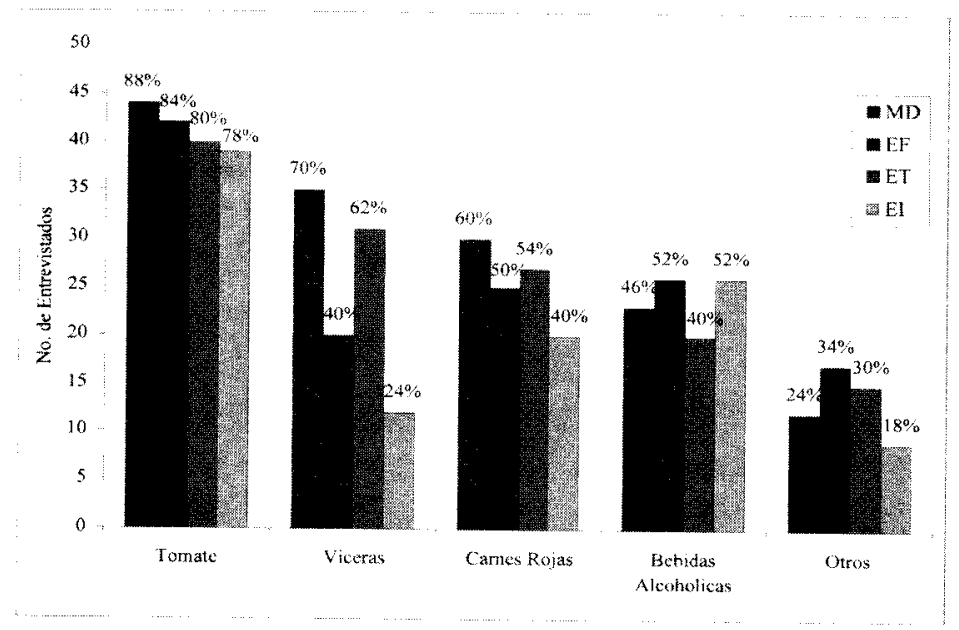

Fuente: Barranco, Jiménez, López. Biolntec XL.III

Un $82.5 \%$ (165/200) de los entrevistados marcaron el tomate como un alimento que elevan el $\mathrm{AU}$, el cual contiene una cantidad muy reducida de proteínas y no podría ser causante de AU elevado. Los alimentos que elevan el AU más destacados fueron las carnes rojas (51\%), las vísceras $(49 \%)$ y las bebidas alcohólicas (47.5\%). Algunos entrevistados decían que el AU era el catabolismo de las proteínas, sin embargo al marcar los alimentos se equivocaban, ya que algunos de los alimentos que marcaban contienen una cantidad muy baja de proteínas, como papa, cebolla, arroz, yuca, agua y pan, contenidas en la categoria de otros..$^{12.14}$

El plato tradicional que identifica la gastronomía dominicana es arroz, habichuelas y carne, acompañado de "frituritas", ensalada y cerveza. De estas, el alcohol disminuye su 
excresión provocando un amuento en sangre, y las carnes y legumbres aumentan su formación por su contenido en proteínas. Es necesario que lo profesionales de la salud dominicana tengan un buen conocimiento sobre el AU ya que la dieta dominicana posee muchos alimentos ricos en proteínas elevadores de la concentración sanguínea del AU.

Esta creencia sobre el AU afecta a la población en varios sentidos: primero, las personas que consideran que el elevado nivel de AU causa vesículas varían su dieta para que no le salgan las vesículas, esto lo hacen innecesariamente porque esas vesículas pueden ser causa de otra pátología, pero no por el AU. ${ }^{3}$ Otra manera que afecta la población es que las personas se someten a una dieta sin tomate, huevo, ni carne y aun así le siguen saliendo las vesículas en las manos, en esos momentos comienzan a ingerir fármacos innecesarios y sin prescripción médica, lo cual puede afectar al paciente con otras complicaciones. Al no tener conocimientos ni poder reconocer correctamente las patologías ligadas al AU, los profesionales de la salud pueden cometer errores de diagnóstico y tratamiento, cometiendo una iatrogenia.

\section{Conclusión}

La investigación determinó que el 2/3 de la población tomada considera que el AU causa lesiones de primer grado como lo son vesículas entre los nudillos, pie de atleta, inflamación de los pies y descamación de las manos, demostrando que tienen un conocimiento erróneo de lo que es el $\mathrm{AU}$, sus manifestaciones y su patología. Al no tener los conocimientos básicos, la tercera parte de las personas creen que algunos alimentos (tomate, cebolla, arroz, otros) elevan los niveles sanguíneos de AU, siendo esto incorrecto, ya que estos alimentos tienen una cantidad reducida de proteínas. ${ }^{12}$ Por 
ejemplo, el $82.5 \%$ de la muestra considera que el tomate causa vesículas en las manos y que al reducir su consume las vesículas desaparecen; esta ignorancia se debe a la mala educación y al entrenamiento deficiente. Esta desinformación es la que relaciona al AU con las lesiones, aunque no tengan nada que ver.

Los estudiantes de inicio tuvieron mayor conocimiento, ya que cerca de las 3/4 partes acertó con la definición de AU. No obstante, estos tuvieron instrucciones recientes sobre el tema, lo que pudo alterar los resultados. Un poco menos de la mitad de las enfermeras acertaron en esta variable dando a conocer la falta de preparación que estas poseen. Las enfermeras escogieron equivocadamente las lesiones que se encontraban en la entrevista, ya que la mitad marcó pie de atleta, la mitad vesículas en los nudillos y cerca de 3/4 partes escogió descamación de las manos. Ningunas mencionaron otra manifestación que pudiera tener el AU. Otros trastornos que la muestra consideró como producto de un nivel elevado de AU fueron problemas de articulaciones y la gota.

El bajo conocimiento del AU se puede deber a la metodología de enseñanza de las universidades las cuales incentivan a las memorizaciones y no a la comprensión clínica de los conocimientos médicos. También puede ser ocasionada por la negligencia de muchos profesionales de la salud, los cuales no se mantienen vanguardistas con las informaciones de las instituciones médicas. Esta y cualquier otra razón no puede ser afirmada por esta investigación debido a que los objetivos no consideraron esta variable.

\section{Recomendaciones}

- Realizar un estudio posterior a este para determinar los factores que han provocado el desconocimiento sobre el 
AU y sus patologías para realizar campañas y seminarios sobre el tema.

- Los médicos y enfermeras deben mantenerse informados y actualizarse con datos vanguardistas para que se elimine la creencia que persiste, porque el $\mathrm{AU}$ no es causante de las lesiones de las cuales se le acusa.

- Debido a la importancia de su trabajo, todos los profesionales de la salud deben entender el metabolismo del AU para no seguir promoviendo esta falsa creencia que esta causando tanto mal a la sociedad.

- Las personas que tengan lesiones en la piel deben realizarse análisis de sangre para determinar el nivel sanguíneo de $\mathrm{AU}$ y consultar con un médico especialista, porque el AU puede que no tenga relación con sus lesiones.

- Antes de someterse a cualquier dieta innecesaria, consultar con un nutricionista para que le indique si es necesario una dieta o si es preferible tomar medicamentos.

- Concienciar y educar mejor a los estudiantes de medicina para que en un futuro no cometan los mismo errores de diagnóstico que cometen los médicos de esta época.

\section{Bibliografía}

1) Mark D, Mark A, et al. Basic Medical Biochemestry a Clinical Approach. Baltimore: William Wilkins, 1996. 64, 565, 631, 634p.

2) Solomon E, Villeé, C, et al. Biología de Ville. México: McGraw-Hill Interamericana, 1998. 979-980, 991-992.

3) Braunwald E, Fauci A, Isselbacher K. Principios de Medicina Interna. México: McGraw-Hill Interamericana, 1998. 2455-2463p

4) San Martín H, J.M. y col: Variación de niveles de ácido úrico en los trastornos hipertensivos del embarazo. Ginecología y Obstetricia de México. México. Feb., 1997; 65. 
5) Rodríguez Pago, C. Hiperuricemia y Gota. http://www.fisterra.com/guias2/gota.htm. España. 2001.

6) National Institute of Arthritis and Musculoskeletal and Skin Diseases: Questions and answers about Gout. http://www.niams.nih.gov/hi/topics/gout/gout.htm. United States. 1999

7) Robbins S, Cotran R, et al. Manual de Patología Estructural y Funcional. $6^{2}$ Ed. Barcelona: Interamericana-McGraw Hill, 2000: 519, 673-674p

8) Medicina Basada en la Evidencia. Gota y seudogota: http://www.msd.es/publicaciones/mmerck_hogar/ seccion_05/seccion_05_052.html. Merck Sharp \& Dohme de España. Madrid, España. 2002

9) La Gota. Enciclopedia de Medicina y Salud. Tomo I. Barcelona: Editorial Sigma S.A., 1994: 310-317p

10) Pérez Ruiz F, Calabozo Raluy M, Herrero Beites A, et al. Análisis de los métodos para clasificar la gota según la excreción renal del ácido úrico. Revista Española de Reumatología. España. 1998; 25(9).

11) Braier L. Fisiopatología y Clínica de la Nutricion: Tomo I. Buenos Aires: Panamericana, 1996: 452-464.

12) Mahan L, Scott-Stump S. Nutrición y Dietaterapia de Kauser. 10 Ed. McGraw Hill Interamericana. México 2001

13) Heinburg D, Weinsier R. Hand Book of Clinical Nutrition. St. Louise: Musbi, 1997: 240.

14) Arroyo, P. Nutriologia Médica. 2a Ed. Buenos Aires: Panamericana, 2001: 535, 289, 339, 435.

15) Safranow K, Korzonek M, Szmatloch E, Rzeuski R, Sulzyc-Bielicka V, et al. Vitamin $C$ and uric acid concentrations in plasma and gastric mucosa in patients regularly consuming vegetables, fruits and fruit juices. Pol Arch Med Wewn 2001 May; 105(5): 383-9. 


\section{ANEXOS}

Anexo $1(\mathrm{~A} 1)$

Entrevista sobre Niveles de conocimiento de Ácido Úrico

Sexo: $F \_M \ldots \quad$ Edad

1- Ocupación:

Doctor/a

Estudiante (carrera)

Enfermero/a

Otros:

2- ¿Sabe usted lo que es el ácido úrico?

Sí__ No

3- Si su respuesta es positiva, defina en sus propias palabras:

4- ¿Todas las personas tienen ácido úrico en el cuerpo?

Sí__. No

5- ¿Considera usted que las altas concentraciones de ácido úrico causa lesiones en la piel?

Sí__ No

6- Si su respuesta fue afirmativa, señale cuales son esas lesiones:

Pie de Atleta

Vesículas entre los nudillos

Inflamación en los Pies

Descamación en las palmas de la mano

Otras

7- ¿Ha sufrido usted de alguna de las lesiones mencionadas?

Sí_ No

8- ¿Cree que la causa fue la alta concentración de ácido úrico?

Sí_ No_ 
Si es positiva ¿Por qué?

9- ¿Se ha sometido a alguna dieta para reducir el nivel de ácido úri$\mathrm{co}$ ?

Sí__ No

10- $i$ Por quién fue recomendada?

Familiar Doctor

Amigo

Usted Mismo

Otros:

11- ¿Cuáles de los siguientes alimentos y bebidas considera usted que eleva las concentraciones de ácido úrico en el organismo?

Hígado

Papas

Espárragos _ - Frutas

Leche - $\quad$ Quesos

Bebidas Alcohólicas __ Huevos

Café Pan

Arroz $\quad$ - L L L L L

Tomate _ C Cebollas

Caldos de Carne _ $\quad$ Espinaca

Sardina $\quad-\quad$ Chocolate

Arenque _ $\quad$ Té

Agua

Yuca

Coliflor

Otros: 